\title{
A new approach to the elucidation of complex chromosome rearrangements illustrated by a case of Rieger syndrome
}

Caroline Mackie Ogilvie, F Lucy Raymond, Rachel H Harrison, Paul N Scriven, Zoe Docherty

Division of Medical and Molecular Genetics, 8th Floor Guy's Tower, Guy's Hospital, London SE1 9RT, UK

C Mackie Ogilvie

F L Raymond

R H Harrison

P N Scriven

Z Docherty

Department of Old

Age Psychiatry and Neuroscience, Institute of Psychiatry, De

Crespigny Park,

Denmark Hill, London

SE5 8AF, UK

F L Raymond

Correspondence to:

Dr Mackie Ogilvie.

Received 4 June 1997 Revised version accepted for publication 25 September 1997

\begin{abstract}
A patient with a complex chromosome rearrangement and unilateral Rieger syndrome is presented. This rearrangement involves four chromosomes and six breakpoints, one of which is at $4 \mathrm{q} 25$, the candidate region for Rieger syndrome. We discuss a novel approach to the elucidation of this case using a multiprobe fluorescence in situ hybridisation method to show rearrangements unpredictable from $G$ banded analysis, and the clear and unambiguous presentation of the karyotype using computer generated colour ideograms.

(F Med Genet 1998;35:234-237)
\end{abstract}

Keywords: complex chromosome rearrangement (CCR); multiprobe fluorescence in situ hybridisation (FISH); computer generated colour ideograms; Rieger syndrome

Complex chromosome rearrangements (CCRs) or "complex chromosome transloca- tions" have been defined" as "those involving more than a reciprocal exchange of segments between two chromosomes", and more recently as "structural rearrangements involving at least three chromosomes and three or more chromosomal breakpoints".

Batista $e t a l^{\beta}$ have reviewed 93 cases of CCR (involving three or more chromosomes); 35 of these were familial, 51 de novo, and in seven cases the families were not studied completely. They concluded that with respect to transmission, subfertility in male carriers, segregation, and parental origin, CCRs follow the same basic principles as simpler rearrangements. The CCRs involved up to seven chromosomes and 10 breakpoints.

CCRs are normally identified in the first instance by standard chromosome banding techniques. However, complete characterisation is not always possible; in these cases, fluorescence in situ hybridisation (FISH, in particular with whole chromosome paints and chromosome specific centromeric and subtelomeric probes) can provide additional
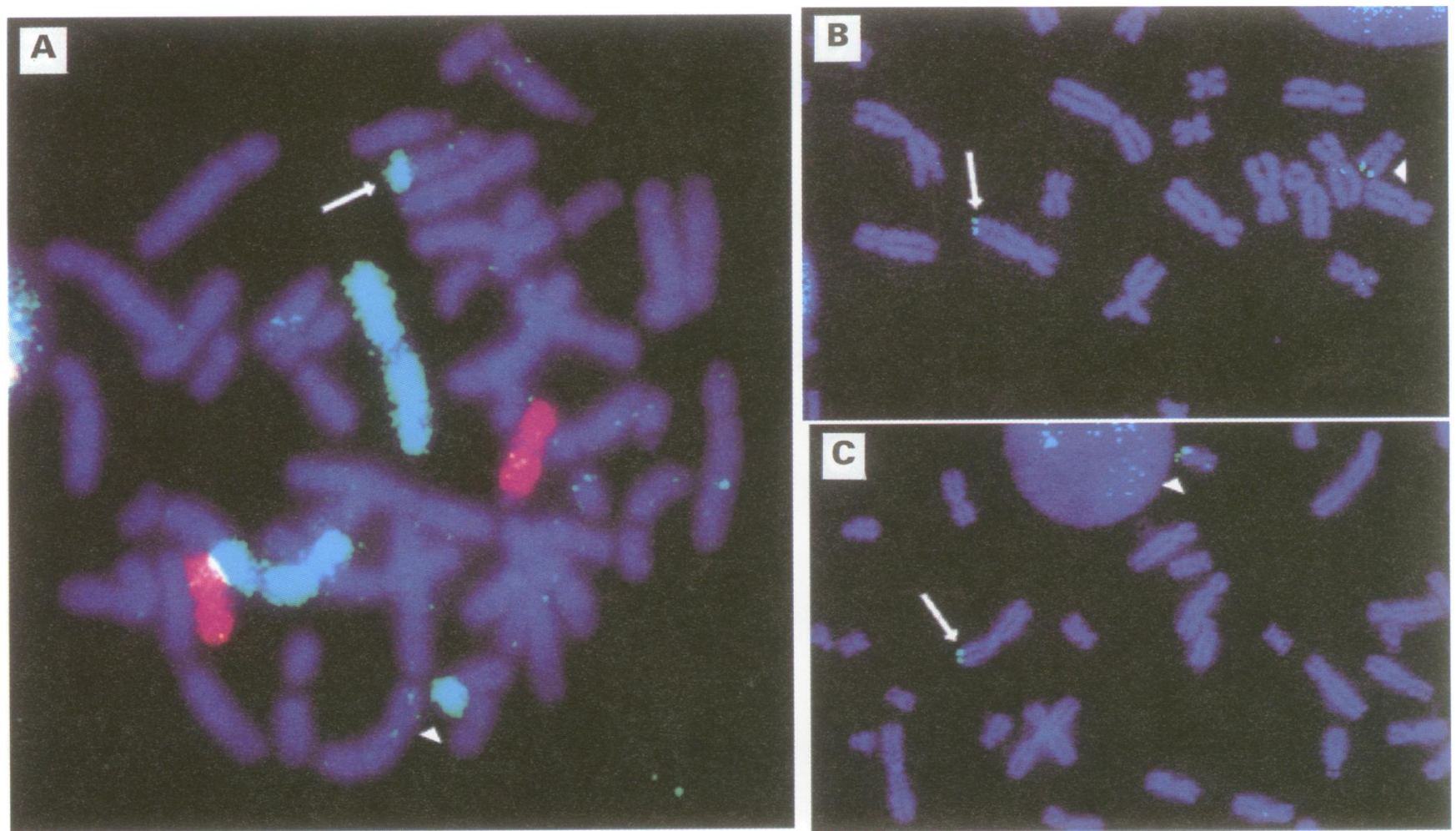

Figure 1 (A) In situ hybridisation of a metaphase spread to whole chromosome paints specific for chromosomes 3 (green) and 13 (red) using the multiprobe FISH procedure; the arrow indicates chromosome 3 material on the derivative chromosome 17 and the arrowhead shows chromosome 3 material on the derivative chromosome 10 . (B) In situ hybridisation with chromosome $17 q$ subtelomeric probe; the arrow shows signal on the derivative chromosome on the derivative chromosome 10. (B) In situ hybridisation with chromosome $17 q$ subtelomeric probe; the arrow shows signal on the derivative chromosom
4 and the arrowhead shows the normal chromosome 17. (C) In situ hybridisation with chromosome $3 p$ subtelomeric probe; the arrow shows the normal chromosome 3 and the arrowhead shows signal on the derivative chromosome 10. The identity of the derivatives in all three images was confirmed by enhancement using PSI Macprobe Powergene software to produce banded chromosomes. 
information resulting in a more accurate definition of the rearrangement. ${ }^{3-6}$

We present a new approach to the elucidation of CCRs using a simple, flexible, and technically straightforward single slide multiprobe FISH procedure ${ }^{7}$ together with computer generated colour ideograms to provide a clear visual interpretation of the structural rearrangements involved. This approach is illustrated using a CCR case presenting with Rieger syndrome.

\section{Case report}

The proband was born after an uneventful twin pregnancy at 38 weeks' gestation by spontaneous vaginal delivery. No abnormalities were detected in the male twin but the proband (female) was found to have a mildly dysmorphic face with a long philtrum and a thin upper lip. The eyes were also abnormal with a congenital cataract of the right eye and a left coloboma. Ophthalmological examination under anaesthetic showed Rieger syndrome with a right sided Peter's anomaly. At the age of 1 year, the right intraocular pressure was raised and she developed buphthalmos. The right eye required enucleation following an injury but at the age of 14 she retains vision (acuity 6/12) in her left eye with normal intraocular pressure and optic disc. Owing to absent dentition, she is currently awaiting surgery to her upper jaw to improve her dental malocclusion and cosmetic appearance. She attends normal school and has reached the same high academic level as her twin brother.

\section{Materials and methods} CYTOGENETICS

Cytogenetic analysis was performed on GTG banded chromosomes from thymidine synchronised cultures of PHA stimulated peripheral lymphocytes, using standard techniques.

\section{IN SITU HYBRIDISATION}

For hybridisation of 24 paints to a single slide, ${ }^{7}$ the slide was first marked with a diamond pen on the underside to form a grid of 12 squares of sides approximately $0.8 \mathrm{~cm}$. A total of $1.5 \mu \mathrm{l}$ of fixed cell suspension was pipetted onto the top surface of the slide into the centre of each square; this typically gave 15-40 metaphase spreads per square, depending on the thickness of the original preparation. The slide was pretreated in $2 \times \mathrm{SSC}(\mathrm{pH} 7.0)$ at $37^{\circ} \mathrm{C}$ for 30
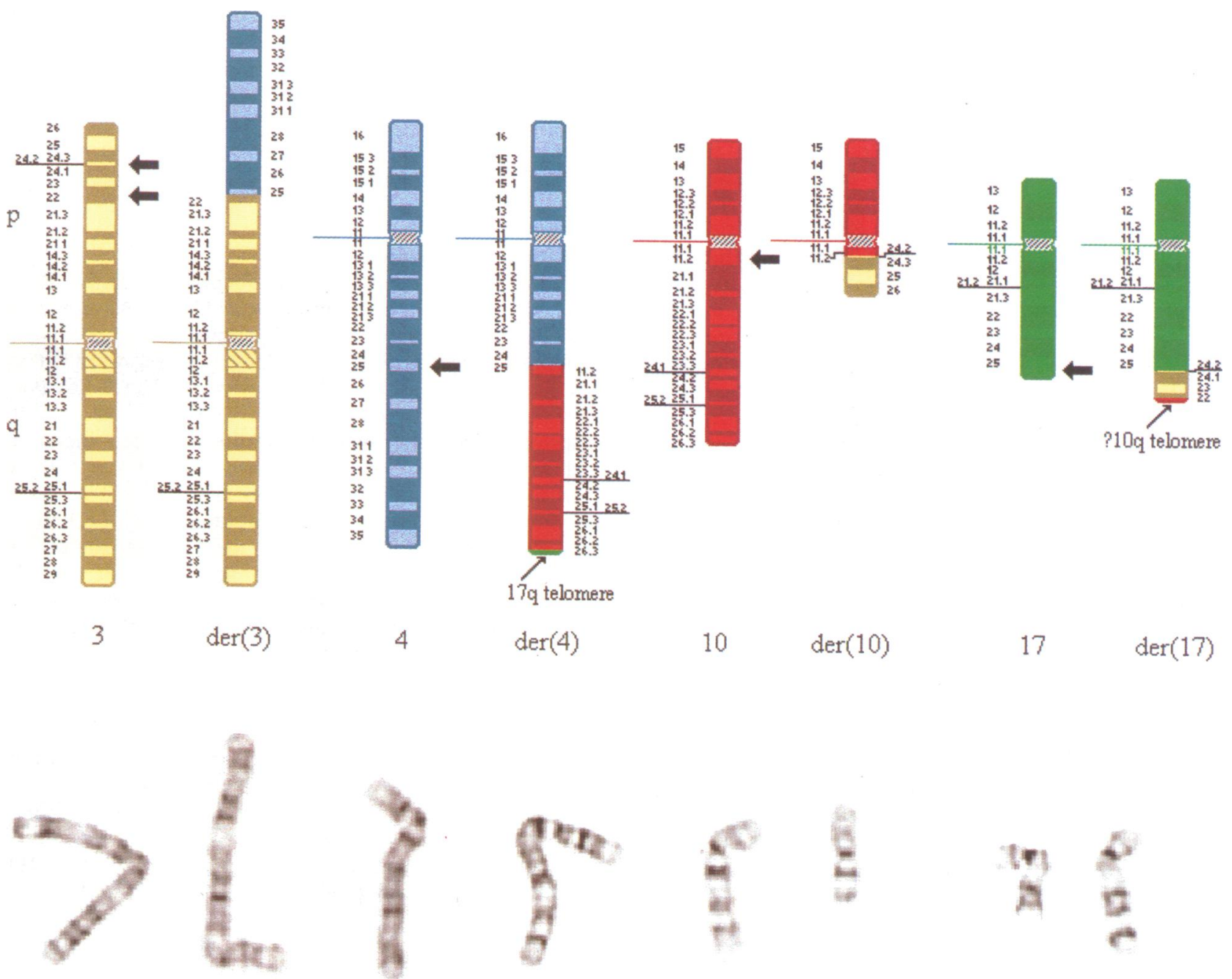

Figure 2 Computer generated colour ideograms with images captured from $G$ banded metaphase spreads. The full ISCN $(1995)^{8}$ description is $46, X X, t(3 ; 10 ; 4)$ ins $(17 ; 3)$ ?t $(10 ; 17)(3 q$ ter $\rightarrow 3 p 22.2:: 4 q 25 \rightarrow 4 q$ ter; 10 pter $\rightarrow 10 q 11.22:: 3 p 24.2 \rightarrow 3$ pter; 4 pter $\rightarrow 4 q 25:: 10 q 11.22 \rightarrow 10 q 26.3:: 17 q 25.3 \rightarrow 17 q$ ter $;$ $17 p t e r \rightarrow 17 q 25.3:: 3 p 24.2 \rightarrow 3 p 22.2::$ ? 10q26.3 $\rightarrow 10 q t e r)$. 
minutes, then dehydrated through an ethanol series $(70 \%, 90 \%, 100 \%$, for two minutes); denaturation was at $70^{\circ} \mathrm{C}$ in $70 \%$ deionised formamide $/ 2 \times \mathrm{SSC}(\mathrm{pH} 7.0)$ for two minutes, followed by dehydration in an ice cold ethanol series (concentrations as above). Biotinylated and FITC labelled whole chromosome paints were obtained from Cambio. Aliquots $(2 \mu \mathrm{l})$ of each of the 24 paints were combined in pairs (one biotinylated with one FITC labelled) in $120.5 \mathrm{ml}$ Eppendorf tubes. After mixing, the paints were denatured by incubation at $70^{\circ} \mathrm{C}$ for two minutes, followed by 30-60 minutes prehybridisation at $37^{\circ} \mathrm{C}$. A rubber solution grid was formed on the denatured, dehydrated slide by the extrusion of Cow Gum through a micropipette tip using a $1 \mathrm{ml}$ disposable syringe. The lines marked on the underside of the slide were followed to ensure that the squares created by the grid contained the fixed chromosome material. Each $4 \mu \mathrm{l}$ mixture of paints was carefully pipetted into the individual squares created by the rubber solution grid. A silicone rubber rim of the same dimensions as the clear glass area of the slide and width 0.2 $\mathrm{cm}$ was applied to the slide with Cow Gum and another slide laid on top. This provided a seal but ensured that the covering slide did not touch the surface of the paints and thus cause mixing of the paints across the top of the grid. Hybridisation was at $37^{\circ} \mathrm{C}$ overnight in a humidified chamber. The covering slide, silicone rubber rim, and rubber solution grid were removed as one piece and stringent washes and detection were according to standard protocols, that is, stringent washes: $65 \%$ formamide $/ 2 \times \mathrm{SSC}(\mathrm{pH} 7.0)$ at $43^{\circ} \mathrm{C}$ for 15 minutes, $2 \times \mathrm{SSC}\left(\mathrm{pH} 7.0\right.$ ) at $37^{\circ} \mathrm{C}$ for eight minutes; detection using the Cambio protocol which entails TR/avidin, $\alpha$-avidin, TR/avidin for the biotinylated paints, in conjunction with a two layer detection system for the FITC labelled paints. Chromosome specific subtelomeric probes were obtained from Oncor and used according to the manufacturer's instructions. (Subtelomeric probes may also be tested using the multiprobe system described above, either alone or in combination with whole chromosome paints.) Chromosomes were counterstained with DAPI in Vectashield and viewed using a fluorescence microscope attached to a PSI MacProbe Image Enhancement system.

\section{Results}

$\mathrm{G}$ banded chromosome analysis of this case initially established a CCR involving chromosomes $3,4,10$, and 17 . It was not clear at this stage, however, whether or not the karyotype was balanced; in addition, the origin of the extra material on the long arm of chromosome 17 could not be determined. The multipaint FISH procedure confirmed the major rearrangements detected on $\mathrm{G}$ banding and identified the extra material on chromosome 17 as originating from chromosome 3 (fig 1A). Material from chromosome 3 was thus present on both the derivative 10 and the derivative 17 . In order to determine which of these derivative chromosomes had the translocated 3pter, chromosome specific subtelomeric probes were tested for chromosomes $3 p$ and $17 q$. The chromosome $3 p$ specific subtelomeric probe hybridised to the normal 3, as expected, and also to the derivative 10 (fig $1 \mathrm{C}$ ), so it was logical to assume that the derivative 17 was the result of an insertional translocation of chromosome 3 material into the long arm of chromosome 17. It was therefore a completely unexpected finding that the $17 \mathrm{q}$ specific subtelomeric probe hybridised to the derivative 4 (fig $1 B$ ), in place of the $10 \mathrm{q}$ telomere. The $10 \mathrm{q}$ specific subtelomeric probe is not available to us, but we assume that it would hybridise to the derivative 17 (fig 2).

The patient's karyotype is therefore balanced and according to standard ISCN $(1995)^{8}$ nomenclature may be written as:

$46, X X, t(3 ; 10 ; 4)$ ins $(17 ; 3)$ ?t $(10 ; 17)(3 q$ ter $\rightarrow 3 p$ $22.2:: 4 q 25 \rightarrow 4$ qter; 10 pter $\rightarrow 10 \mathrm{q} 11.22:: 3 \mathrm{p} 24.2$ $\rightarrow 3$ pter;4pter $\rightarrow 4 \mathrm{q} 25:: 10 \mathrm{q} 11.22 \rightarrow 10 \mathrm{q} 26.3::$ $17 q 25.3 \rightarrow 17 q t e r ; 17$ pter $\rightarrow 17 q 25.3:: 3 p 24.2 \rightarrow$ 3p22.2::?10q26.3 $\rightarrow 10$ qter). This may also be described in a slightly more concise form as: $46, \mathrm{XX}, \operatorname{der}(3,4,10,17) \mathrm{t}(3 ; 10 ; 4)(\mathrm{p} 22.2 ; \mathrm{q} 11.22$; $\mathrm{q} 25)$ ins $(17 ; 3)(\mathrm{q} 25.3 ; \mathrm{p} 24.2 \mathrm{p} 22.2)$ ? $\mathrm{t}(10 ; 17)$ (q26.3;q25.3).

Parental karyotypes were normal on G banded chromosome analysis; FISH with the $17 q$ subtelomeric probe showed signal on the terminal region of the two homologues of chromosome 17 in both parents, indicating that the unexpected subtelomeric rearrangement in the proband did not originate from a parental subtelomeric translocation between chromosomes 10 and 17.

\section{Discussion}

The mechanism of formation of CCRs is unclear; cases must by definition have at least three breakpoints, but more breakpoints are usual, and two cases have been reported with as many as $10 . .^{5}$ In cases where FISH has been used for re-evaluation of $G$ banded analysis, this has usually resulted in an increased estimate of the number of breaks. ${ }^{340}$ However, the probes chosen for testing are usually determined by information from $G$ banded analysis concerning the chromosomes involved. The case presented here underlines the need for multiprobe analysis of these cases, as some of the rearrangements involved may be unpredictable. The availability of both a commercial product (Cytocell Ltd) and the fast, cheap, and technically straightforward method described here means that either may be used to test the complete set of chromosome paints. It is our understanding that complete sets of chromosome specific subtelomeric probes will also be available commercially in the near future, and we would recommend that these are applied, using a multiprobe technique, to uncover cryptic subtelomeric translocations. The more complex a rearrangement, the higher would be the expected proportion of unbalanced gametes produced at meiosis. A full characterisation of complex rearrangements is therefore important for a more precise estimate of risk of progeny with unbalanced 
karyotypes and is absolutely essential for accurate prenatal diagnosis.

Both alternative forms of karyotype described above are cumbersome and difficult for the patient and clinician to understand. In addition, the standard ISCN conventions for complex karyotypes are ambiguous (a point also made by others ${ }^{5}$ ) and may result in different interpretations by different centres. The ISCN therefore recommends either the illustration of complex rearrangments or verbal descriptions thereof; visual representation is clearly essential in such a complicated case as that described here. Previous authors have either used partial karyotypes ${ }^{5}$ or black and white images, cut and pasted, either with breakpoints indicated with arrows and band numbers $^{35}$ or coloured in to represent the rearrangements. ${ }^{6}$ The computer generated colour ideograms (CGCIs) presented here (fig 2) are clear and simple, and are easily manipulated to represent most chromosome rearrangements. This department uses them routinely when reporting translocations, inversions, and insertions, as well as CCR cases. It is also our policy to provide clinicians with CGCI representations of the possible outcomes of pregnancies for patients with balanced rearrangements.

Interestingly, there are several published case reports of patients with Rieger syndrome who also have interstitial deletions in the region $4 \mathrm{q} 25-4 \mathrm{q} 27^{11} 12$ or balanced translocations involving the $4 \mathrm{q} 25-26$ region. ${ }^{12-14}$ The case presented here therefore lends further support to $4 \mathrm{q} 25$ as a candidate region for the gene involved in Rieger syndrome. We would like to acknowledge Dr H Issler, Dr C Garrett, and Mr R Wormald for their clinical involvement with the patient. Dr F L Raymond is funded by a Wellcome Clinical Training
Fellowship, and FISH imaging equipment was purchased using a grant from the Special Trustees of Guy's Hospital.

1 Pai GS, Thomas GH, Mahoney W, Migeon GR. Complex chromosome rearrangements. Clin Genet 1980;18:436-44.

2 Gorski JL, Kistenmacher ML, Punnett HH, Zackai EH, Emanuel BS. Reproductive risk for carriers of complex Med Genet 1988;29:247-61.

3 Batista DAS, Pai GS, Stetten G. Molecular analysis of a complex chromosomal rearrangement and a review of famplial cases. Am f Med Genet 1994;53:255-63.

4 Batista DAS, Tuck-Muller CM, Martinez JE, Kearns WG, Pearson PL, Stetten G. A complex chromosomal rearPearson PL, Stetten G. A complex chromosomal rearrangement detected prenatally and studied by fluo

5 Spikes AS, Hegmann K, Smith JL, Shaffer LG. Use of fluorescence in situ hybridization to clarify a complex chromosomal rearrangement in a child with multiple congenital anomalies. Am $\mathcal{F}$ Med Genet 1995;57:31-4.

6 Wallerstein R, Gibas L, Anderson CE, Jackson L. Diagnosis of a complex chromosomal rearrangement using fluorescent in situ hybridisation. F Med Genet 1996;33:793-4.

7 Mackie Ogilvie C, Waddle K, Mandeville J, Seller MJ, Docherty Z. Rapid identification of multiple supernumerary ring chromosomes with a new FISH technique. fMed Genet 1997;34:912-16.

8 ISCN (1995). In: Mitelman F, ed. An international system for human cytogenetic nomenclature. Basel: Karger, 1995.

9 Tupler R, Maraschio P, Gerardo A, Mainieri R, Lanzi G Tupler R, Maraschio P, Gerardo A, Mainieri R, Lanzi G,
Tiepolo $L$. A complex chromosome rearrangement with 10 breakpoints: tentative assignment of the locus for Williams breakpoints: tentative assignment of the locus for William
syndrome to $4 \mathrm{q} 33 \rightarrow \mathrm{q} 35.1$. F Med Genet 1992;29:253-5.

syndrome to 4q33 $\rightarrow \mathrm{q} 35.1$. F Med Genet 1992;29:253-5.
10 Mercier S, Fellmann F, Cattin J, Bresson JL. Molecular analysis by fluorescence in situ hybridization of a prenatally detected de novo complex chromosome rearrangement t(2q;3p;4q;13q). Prenat Diagn 1996;16:1046-50

11 Vaux C, Sheffield L, Keith CG, Voullaire L. Evidence that Rieger syndrome maps to $4 \mathrm{q} 25$ or $4 \mathrm{q} 27$. F Med Genet 1992;29:256-8.

12 Flomen RH, Gorman PA, Vatcheva R, et al. Rieger syndrome locus: a new reciprocal translocation $t(4$; 12)(q25;q15) and a deletion $\operatorname{del}(4)$ (q25q27) both break between markers D4S2745 and D4S193. F Med Genet 1997;34:191-5.

13 Makita Y, Masuno M, Imaizuni S. Rieger syndrome with de novo reciprocal translocation $\mathrm{t}(1 ; 4)(\mathrm{q} 23.1 ; \mathrm{q} 25)$. Am $\mathcal{f}$ Med novo reciprocal transloce

14 Murray JC, El-Shanti H, Lindgren C, et al. Combined mapping approaches to Rieger syndrome. Am $\mathcal{F}$ Hum Gene Suppl 1993;53:A141. 